\title{
Human genetic risk of treatment with antiviral nucleoside analog drugs that induce lethal mutagenesis - the special case of molnupiravir
}

Michael D Waters ${ }^{1}$, Stafford Warren ${ }^{2}$, Claude Hughes ${ }^{3}$, Philip Lewis ${ }^{4}$, and Fengyu Zhang ${ }^{5}$

${ }^{1}$ Michael Waters Consulting USA

${ }^{2}$ Anne Arundel Medical Center

${ }^{3}$ Duke University Medical Center

${ }^{4}$ Cornell University

${ }^{5}$ Global Clinical and Translational Research Institute

November 4, 2021

\section{Hosted file}

Authorea Preprint Submission on 3 Nov 2021.pdf available at https://authorea.com/users/ 444231/articles/544190-human-genetic-risk-of-treatment-with-antiviral-nucleoside-analogdrugs-that-induce-lethal-mutagenesis-the-special-case-of-molnupiravir 